\title{
Dimensions éthiques de la dynamique des modèles de consommation alimentaires ${ }^{*}$
}

\author{
Soraya DUBOC ${ }^{1}$ \\ Marie DE LATTRE-GASQUET ${ }^{2}$ \\ ${ }^{1}$ Membre du comité consultatif \\ commun d'éthique pour la recherche \\ agronomique, \\ ingénieur agroalimentaire \\ 2 Secrétaire du comité consultatif \\ commun d'éthique pour la recherche \\ agronomique - CIRAD, \\ 42 rue Scheffer, \\ 75116 Paris \\ $<$ marie.de_lattre-gasquet@cirad.fr $>$
}

Article reçu le 19 juillet 2012

Accepté le 27 juillet 2012

\section{Les modèles \\ de consommation \\ s'inscrivent dans un contexte \\ d'interdépendance \\ mondialisée avec \\ des incertitudes \\ majeures \\ et des contraintes \\ croissantes}

La dynamique des modèles

de consommation : plus

de menaces que d'opportunités

sur un objet aux contours flous

Pour satisfaire les besoins physiologiques, conjuguer santé, plaisir et partage, les aliments peuvent être préparés et combinés de multiples manières, en

\begin{abstract}
Ethical dimensions of the dynamics of food consumption models Consumption patterns are part of a globalized context of interdependence with major uncertainties and increasing constraints. Linking them with food security is essential. Challenges are therefore political, economic, environmental, social but also ethical. Ethics is, mainly, a reflexive intellectual attitude based on problematisation and questioning. One must questions one's own actions and relationships with others, both in intention and impact. With regard to consumption patterns and food security, the areas in which "concern [should be] necessary and endless" are numerous. Nine major ethical issues can be stated; they are related to agricultural production, consumption, traceability and research priorities.
\end{abstract}

Key words: globalization, food consumption models, food security, ethics

fonction de facteurs d'ordre culturel, social, géographique, historique. La sociologie donne à cet ensemble l'expression de modèles alimentaires. "Les modèles alimentaires sont un corps de connaissances technologiques, accumulées de génération en génération, permettant de sélectionner des ressources dans un espace naturel, de les préparer pour en faire des aliments, puis des plats, et de les consommer. Mais ils sont en même temps des systèmes de codes symboliques qui mettent en sciene les valeurs $d^{\prime} u n$ groupe humain participant à la construction des identités culturelles et aux processus de personnalisation. [...] Les contraintes de la mécanique biologique sont [donc] relativement lâches et lui laissent un vaste espace de liberté dans lequel le social et le culturel peuvent jouer sans conséquences vitales déterminantes, pour créer à la fois de l'identité et de la différenciation sociale. "(Poulain, 2002). Deux modèles alimentaires coexistent dans le monde (Fischler, Masson, 2007) : le premier est individualiste et nutritionniste (il s'agit d'incorporer les bons nutriments plutôt que de manger) où il appartient à chacun de faire les bons choix. II en découle une culpabilité, parce qu'il est à peu près impossible de faire les bons choix en permanence. Un deuxième modèle accorde au contraire plus d'importance à l'aspect convivial de I'alimentation. Bien manger, c'est partager un repas avec d'autres, et donc soumettre son appétit à des règles collectives, avec une notion de plaisir très présente, plaisir du partage et plaisir gustatif (Chabrol, 2008).

Le développement économique et les évolutions des conditions de vie ont entraîné des changements majeurs dans la consommation alimentaire, favorisés principalement par l'essor de l'industrie alimentaire, le développement de la grande distribution et l'extension de la restauration hors foyer. L'offre alimentaire est également élargie par l'intensité des échanges internationaux de denrées

\footnotetext{
*Cet article reprend en grande partie le texte d'un avis du Comité consultatif commun d'éthique pour la recherche agronomique. Avis sur "Sécurité alimentaire et modèles de consommation alimentaire ". INRA et CIRAD. Mars 2010. http://www.cirad.fr/qui-sommes-nous/le-cirad-enbref/organisation/instances-et-comites/comite-consultatif-commun-d-ethique
}

Pour citer cet article : Duboc S, de Lattre-Gasquet M. Dimensions éthiques de la dynamique des modèles de consommation alimentaires. OCL $2012 ; 19(5): 270-275$. doi : 10.1684/ocl.2012.0469 
agricoles et de produits transformés. Dans les pays développés, la part de I'alimentation dans le budget disponible des ménages a fortement baissé : en France, elle est passée de $50 \%$ en 1950 à une moyenne de $15 \%$ dans les années 2000. D'un point de vue nutritionnel, les changements sont caractérisés d'abord par un accroissement de la ration calorique par tête puis par un changement radical de la structure du régime alimentaire avec une baisse de la consommation de produits céréaliers et de féculents et une augmentation des matières grasses, des sucres rapides et des produits d'origine animale. Cette évolution observée dans les pays développés se généralise progressivement aux pays émergents même si des différences apparaissent entre les pays en particulier ceux dont les cultures alimentaires font une large place aux produits végétaux (Inde, Japon, etc.) (Esnouf et al., 2011).

Pour tous les acteurs, mais en particulier ceux du domaine des corps gras, il devient utile de réfléchir à ses responsabilités et à ce qui pourrait être universel dans les modèles alimentaires, et ce qui pourrait être spécifique. Quelques pistes :

- ce qui pourrait être universel : l'équilibre nutritionnel (en tenant compte cependant de l'âge, du sexe, de la taille, de la corpulence, des conditions physiques et de l'état physiologique, du mode de vie), la durabilité, en termes d'utilisation de ressources locales (souveraineté alimentaire), la nonvolatilité des prix et la stabilité de la disponibilité des aliments, l'accès à la nourriture ;

- ce qui doit être spécifique : les aspects culturels, l'agencement des repas, les recettes, le type d'aliments associés (dans une gamme permettant l'équilibre nutritionnel), le terroir, les modes de distribution et de production, les manières de table. .

Même si le choix alimentaire est individuel, il se trouve induit ou conditionné par une série de facteurs dont les principaux sont d'ordre biologique (faim, soif, satiété, qualités sensorielles), social (culture, famille, pairs, structuration des repas), économique (coût, revenus, disponibilité des aliments), physique (accès), temporel (préparation), psychologique ou cognitif (éducation nutritionnelle, informations). Les produits consommés, les pratiques et les représentations associées dessinent ainsi des modèles de consommation qui, aujourd'hui, apparaissent peu formalisés. La régulation complexe des systèmes alimentaires, dont les acteurs sont multiples, rend d'autant plus difficile la mise en place de leviers $d^{\prime}$ action. En effet, l'une des spécificités du domaine alimentaire est d'être au carrefour des choix individuels et d'une très grande diversité d'acteurs socioéconomiques.

\section{Vers des changements majeurs dans le rôle des acteurs et leur environnement?}

Les systèmes alimentaires sont influencés par une multiplicité $d^{\prime}$ acteurs dont I'implication évolue fortement sous l'effet du contexte socio-économique et environnemental :

- les consommateurs qui tentent de s'organiser ;

- les opérateurs privés: agriculture d'une part, et d'autre part industrie alimentaire et distribution, qui ont désormais une influence très significative sur le système alimentaire ;

- les États ou les ensembles régionaux, par le biais des politiques agricoles (Politique Agricole Commune, Farm Act, Plan National pour I'Alimentation en France ou ses équivalents dans d'autres pays), des organismes de normalisation, mais aussi de leur politique énergétique, qui enrôle l'agriculture dans la production de biocarburants.

Ces acteurs agissent dans un environnement marqué par la raréfaction des ressources en eau et en sols, la volatilité des prix alimentaires (Daviron, 2012), l'apparition de pratiques spéculatives à grande échelle sur le foncier, l'impératif de protection de l'environnement, le réchauffement climatique qui rend plus critique les deux facteurs précédents, et le progrès scientifique et technique.

Les politiques agricoles, ainsi que les progrès scientifiques et techniques, ont permis d'atteindre l'autosuffisance, dans la plupart des pays de I'OCDE, au prix d'une pression forte sur les écosystèmes, ce que la société dans son ensemble ne cautionne plus. Parallèlement, une succession de crises sanitaires (vache folle, grippe aviaire, etc.), de pratiques frauduleuses (dioxine, colorants interdits, huiles frelatées, mélamine) et les controverses autour des OGM ont développé une certaine méfiance du consommateur envers les opérateurs de la chaîne alimentaire, et ce, malgré des réglementations, européenne et internationale, de plus en plus attentives à encadrer les différentes filières à risques existants ou émergents, ou très soucieuse $d^{\prime}$ une meilleure information du consommateur. La réglementation relative aux allégations nutritionnelles et de santé ${ }^{1}$ ainsi que la nouvelle réglementation sur l'étiquetage des denrées alimentaires (Règlement UE $\mathrm{n}^{\circ}$ 1169/2011 du 25 octobre 2011) en témoignent. Cette dernière réglementation prévoit, entre autres, que la nature exacte des matières grasses végétales (palme, coco, etc.) soit indiquée de façon claire dans la liste des ingrédients; par ailleurs, sur le tableau nutritionnel, à compter de décembre 2014, les " graisses » devront être déclarées juste après la valeur énergétique (alors qu'aujourd' hui, les lipides sont en troisième position des nutriments déclarés, après les protéines et les glucides).

Une production raisonnée, respectueuse des droits sociaux et de l'environnement, avec une certaine efficacité économique, telle semble se dessiner l'attente d'un nombre croissant de citoyens dans les pays développés surtout, et de façon croissante dans les pays émergents. Les associations de consommateurs cherchent à défendre les intérêts des populations et à peser sur les décisions politiques.

Les entreprises alimentaires (producteurs, distributeurs) agissent pour informer et rassurer les consommateurs et les décideurs politiques. Elles doivent de plus en plus faire la preuve de l'exercice effectif de leur responsabilité sociale : respect des consommateurs, y compris des plus vulnérables, contribution à la santé et au bien-être, respect des droits sociaux et de l'environnement dans leurs stratégies d'approvisionnement, de communication commerciale et de développement à long terme. Des systèmes de certification se mettent en

\footnotetext{
${ }^{1}$ Comme le prévoit le règlement (CE) $n^{\circ} 1924$ /2006, la liste des allégations nutritionnelles et de santé a été validée en 2012. Depuis le 16 mai 2012, le registre interactif des 222 allégations autorisées à compter de décembre prochain a été mis en ligne par la Commission européenne au terme d'un processus d'évaluation démarré en 2008.
} 
place pour refléter ces nouveaux engagements envers des parties prenantes négligées jusqu'à récemment, et constituent des éléments de régulation sociopolitique (Hartlieb et Jones, 2009).

Comment jusqu'à présent la recherche agronomique a-t-elle répondu aux défis de la sécurité alimentaire et des modèles de consommation? Le bilan est contrasté $d^{\prime}$ 'après les travaux menés par I'Office parlementaire d'évaluation des choix scientifiques et technologiques (OPECST, 2004) et les résultats de l'évaluation internationale des sciences et technologies agricoles pour le développement (IAASTD, 2009a). II est difficile de déterminer l'impact de la recherche en général sur les modèles alimentaires, faute d'éléments d'évaluation, mais aussi parce que les modèles alimentaires évoluent sous l'influence de multiples facteurs, qu'il convient de mieux cerner. La réflexion stratégique "duALIne » (Durabilité de I'ALImentation face à de Nouveaux Enjeux) menée par I'INRA et le CIRAD contribue à analyser les tendances majeures d'évolution des systèmes alimentaires pour en déduire les pistes de recherche prioritaires pour les communautés scientifiques nationales et internationales (Esnouf et al., 2011).

L'ensemble de ces évolutions conduit à envisager pour la recherche agronomique de nouvelles raisons d'agir en prenant en compte des demandes de la société civile et des institutions, en vue de contribuer à une sécurité alimentaire universelle et perpétuelle.

\section{On ne peut pas penser aux modèles de consommation alimentaire sans réfléchir à la sécurité alimentaire, question qui inclut l'obésité}

Plus d'un milliard de personnes souffrent encore de la faim dans le monde avec une grande diversité de situations. Les impacts de la fluctuation des cours mondiaux sur la sécurité alimentaire et la nutrition des ménages dépendent du produit concerné et des politiques que mènent les pays (FAO, 2011) et il y aura près de 700 millions d'obèses en 2015 selon l'OMS (OMS, 2006). Dans les pays développés, se nourrir pose un problème financier pour une part croissante de la population, problème amplifié par la crise économique du début des années 2000 : personnes marginalisées, travailleurs pauvres, personnes âgées aux revenus insuffisants (Laisney et Soyeux, 2009). Dans les pays pauvres ou en voie de développement, les consommateurs urbains sont fortement touchés par la forte augmentation des prix des produits alimentaires et les agriculteurs ne sont pas à l'abri de ces difficultés économiques (ils représentent près des trois quarts de la population souffrant de la faim). Par ailleurs, les consommateurs à faible pouvoir d'achat privilégient des produits à bas prix au détriment de l'équilibre alimentaire. L'obésité constitue aujourd'hui un signe d'insécurité alimentaire et un marqueur social de la pauvreté dans les pays " riches" et dans les pays en voie de développement, où elle se répand, y compris dans certains pays où sévit la faim.

Ainsi, alors que pendant le demi-siècle écoulé, " la nourriture est devenue moins chère et la disponibilité calorique moyenne a augmenté (au milieu des années $60,57 \%$ de la population mondiale vivait dans des pays où la disponibilité calorique moyenne était inférieure à $2200 \mathrm{kcal}$; aujourd'hui, la proportion est de $10 \%)$ ) (IAASTD, 2009b), la sécurité alimentaire est loin d'être assurée pour près d'un habitant sur deux si l'on prend en compte les personnes atteintes de surcharge pondérale ou d'obésité.

Le droit à la nourriture n'est pas un concept nouveau ; il est déjà présent dans la Déclaration universelle des droits de l'homme adoptée par l'Assemblée générale des Nations Unies en 1948. Actuellement, ce droit à la nourriture est reconnu dans la constitution de plus de 40 pays et pourrait être inscrit dans le droit judiciaire de quelque 54 pays (McClain-Nhlapo, 2004).

Les concepts relatifs à la sécurité alimentaire ont évolué parallèlement à l'évolution de la pensée politique officielle (FAO, 2006). Le terme est apparu au milieu des années soixante-dix, lorsque le Sommet mondial de l'alimentation a défini la sécurité alimentaire en termes d'approvisionnement : garantir la disponibilité et la stabilité des prix des produits alimentaires de base à l'échelon national et international. La théorie de la famine d'Amartya Sen (Sen, 1981) a mis l'accent sur les droits et les capacités des individus à échanger et en 1983, la notion d'accès physique et économique aux denrées alimentaires a été introduite. En 1986, un rapport de la Banque Mondiale introduit les distinctions entre l'insécurité alimentaire chronique, associée à des problèmes de pauvreté permanente ou structurelle et à de faibles revenus, et I'insécurité alimentaire transitoire liée à des périodes particulièrement critiques résultant $d^{\prime}$ une catastrophe naturelle, d'un marasme économique ou d'un conflit. La définition du Sommet mondial de I'alimentation de 1996 est celle encore admise aujourd'hui : " $L a$ sécurité alimentaire existe lorsque tous les êtres humains ont, à tout moment, un accès physique et économique à une nourriture suffisante, saine et nutritive leur permettant de satisfaire leurs besoins énergétiques et leurs préférences alimentaires pour mener une vie saine et active".

Les qualificatifs " saine " et " nutritive " renvoient à l'aspect sanitaire (Food Safety) des aliments disponibles. Cette définition de la sécurité alimentaire (Food Security) couvre les fonctions essentielles de I'alimentation - sociale (et identitaire), biologique et hédonique - qu'elle considère comme indissociables. La sécurité alimentaire est un préalable pour que toute personne puisse envisager une vie digne.

Le défi alimentaire est d'assurer cette sécurité alimentaire dans des conditions durables. Les enjeux sont d'ordres non seulement éthiques, mais aussi politiques, économiques, sociaux et environnementaux.

\section{Principaux problèmes éthiques liés aux dynamiques des modèles de consommation alimentaire}

L'éthique est, pour l'essentiel, une attitude intellectuelle réflexive fondée sur la problématisation et le questionnement. Questionnement sur ses propres actions, ses relations avec autrui aussi bien dans leur intention que leur impact. La place de I'homme dans l'univers, sa capacité et sa légitimité à modifier la nature par ses interventions sont des interrogations très anciennes. Au cours des siècles, en Occident, mais aussi dans toutes les grandes cultures à travers le monde, plusieurs critères de questionnement ont été proposés, souvent par des philosophes. 
S'agissant des modèles de consommation et de la sécurité alimentaire, les domaines dans lesquels "l'inquiétude [doit être] nécessaire et sans fin " (Sicard, 2006) sont nombreux. Neuf questions éthiques majeures peuvent être énoncées, qu'elles soient en lien avec la production agricole, la consommation, la traçabilité ou les thématiques de recherche. Elles sont particulièrement importantes pour les acteurs du domaine des corps gras.

\section{En lien avec la production amont}

L'intensification de l'agriculture conduit à des émissions de gaz à effet de serre, des déchets, à la pollution des sols et des eaux, à la déforestation, ainsi qu'à la présence non intentionnelle de substances chimiques indésirables dans les aliments. Pourtant, il est nécessaire d'augmenter le volume de production agricole de l'ordre de $70 \%$ pour nourrir les 9 milliards d'habitants à I'horizon 2050 selon la FAO (2009).

Dans son avis «Éthique et intensification agricole durable », la FAO (FAO, 2004) souligne que I'intensification n'a pas la même justification suivant le cadre de pensée utilisé. Le modèle utilitariste lui trouve une justification dans la croissance démographique; dans l'optique des droits, une intensification qui exclut de la production certains groupes est inadmissible. La FAO recommande, en ce qui concerne les projets d'intensification, "de formuler une éthique qui emprunte à chacune des traditions pouvant servir à déterminer et à peser les responsabilités morales. En omettre ne serait-ce qu'une compromet la capacité de formuler, d'analyser et de remplir les obligations morales qui peuvent surgir en rapport avec la croissance des populations et avec les besoins d'intensification agricole qui en résulteront. "

\section{En lien avec les questions d'ordre socio-culturel et de consommation}

\section{Problèmes éthiques liés à l'accès à la nourriture, notamment pour les plus démunis}

Les problèmes éthiques liés à l'accès à la nourriture, au commerce, sont nombreux. Cette question a fait l'objet d'études, notamment celle du Comité économique et social européen (CESE, 2005). Par exemple, des travaux peuvent porter sur les manières de concilier une alimentation à un prix abordable pour le consommateur avec la nécessité de prix aux producteurs comparativement plus élevés que ceux des produits importés, notamment quand ces producteurs sont des paysans pauvres. Comment concilier des prix bas et une alimentation sans trop de corps gras ou de sucres ? Quelle est la chaîne de valeur pour les produits de base? Comment sont mobilisées les expériences qui ont réussi, et celles qui ont échoué : quelles sont les causes des succès et des échecs?

\section{Questionnements sur la pertinence et la faisabilité d'un modèle alimentaire unique}

L'alimentation étant aussi un fait social et culturel, il est naturel que différents modèles de consommation coexistent ; ils apportent à des populations données une satisfaction en rapport avec l'utilisation de ressources locales. Cependant, trois mécanismes semblent pousser à I'adoption d'un modèle alimentaire unique: le processus de distinction sociale, le mythe égalitaire et la montée de la pensée hygiéniste (Poulain, 2002). Pour autant, la tentation d'appliquer un modèle alimentaire unique $n^{\prime} a$ guère de sens. Cela reviendrait à abandonner des ressources locales qui ne seront pas aisément remplaçables et dévaloriserait les agriculteurs traditionnels. Le maintien d'un niveau élevé de diversité alimentaire répond à différents enjeux, au premier rang desquels celui de la dignité (respect des choix de groupes sociaux, choix qui sont souvent facteurs de cohésion); à cela il convient d'ajouter la complexité des situations et des réalités socio-économiques, mais aussi la nécessité d'assurer robustesse, adaptabilité et résilience ${ }^{2}$ aux environnements de vie. C'est au prix de la diversité que la sécurité alimentaire à long terme pourra être assurée.

Mais il est certain que les institutions publiques et les entreprises propagent, à la fois consciemment et inconsciemment, des modèles alimentaires fondés sur l'état de leurs recherches et leurs propres priorités. Or, encourager un modèle alimentaire affecte l'organisation sociale et économique.

\footnotetext{
${ }^{2}$ La résilience est la capacité d'un écosystème ou d'une espèce à récupérer un fonctionnement et/ ou un développement normal après avoir subi un traumatisme.
}

Problèmes éthiques liés aux modèles de consommation respectueux

des "écosystèmes" culturels

Une alimentation suffisante et adéquate doit respecter les modèles culturels, tout en évitant l'écueil qui consisterait à enfermer certains groupes sociaux dans des pratiques qui résultent des contraintes du milieu ou d'habitudes devenues une seconde nature. Il s'agira de mettre en évidence les conditions pour permettre un choix éclairé par les personnes ou les groupes sociaux concernés.

\section{Problemes éthiques liés \\ à la surconsommation de produits gras, de sucres}

Il est établi que la croissance du revenu s'accompagne d'une très forte augmentation de la part des lipides (seuls les lipides $d$ 'origine végétale régressent), d'une baisse de la part des glucides (l'accroissement de la consommation des produits sucrés ne compensant pas la baisse de la consommation des céréales) et enfin d'une stabilité de la part des calories protéiques. Sachant cela, comment accompagner les changements de régime alimentaire dans les pays en développement et dans les pays développés pour éviter les conséquences néfastes sur la santé humaine et la pression sur les ressources agricoles?

\section{Problèmes éthiques liés à I'aide alimentaire, nécessairement ponctuelle et non structurelle}

L'aide d'urgence est une exigence morale mais elle ne sera jamais suffisante. La pérennisation de l'aide alimentaire est problématique car elle maintient dans I'assistanat les personnes concernées, sans leur permettre de développer leurs " capabilités " (Sen, 2000) par la formation, l'éducation et surtout l'exercice de leurs responsabilités. II peut paraître légitime et souhaitable que la recherche intervienne dans des situations d'aide alimentaire, afin qu'elle en tire des enseignements pour son domaine d'action.

\section{Problèmes éthiques liés à la sécurité sanitaire des aliments et à la traçabilité des produits}

Les responsables de la gestion qui réalisent I'analyse des risques font rarement connaître ouvertement leurs jugements de valeur et leurs choix éthiques. La FAO (FAO, 2002) propose cinq groupes de valeurs comme base à toute recommandation en matière de sécurité 
sanitaire : le droit à une alimentation adéquate, la confiance, l'optimisation, le consentement informé et l'équité.

Le Conseil National de I'Alimentation a appelé l'attention des pouvoirs publics sur le fait que la traçabilité peut modifier très sensiblement les rapports entre les consommateurs et I'aliment, dès lors qu'il est matériellement possible de mieux connaître "l'histoire » d'un produit sans avoir nécessairement à entreprendre des recherches très approfondies (CNA, 2001). II a insisté sur le fait que les possibilités techniques nouvelles pourraient développer davantage les attentes relatives à I'environnement ou à l'éthique. Quelles recherches sont menées dans ce domaine? Quel est le degré effectif de la protection fournie au consommateur par un système de traçabilité ? Ne protège-t-on que le consommateur: qui sont les bénéficiaires effectifs, qui sont les éventuels perdants dans de telles démarches?

\section{En lien avec la production de connaissances et de savoirs}

Problèmes éthiques liés à l'équilibre entre les bénéficiaires de la recherche Comment les institutions publiques de recherche et les entreprises privées équilibrent-elles leurs efforts de recherche entre le producteur/le fabricant et le consommateur ? Étant donné l'ampleur des défis et l'obligation morale de libérer de la faim et de mauvaises pratiques alimentaires une part importante de la population mondiale, sur quels sujets faire porter les priorités de recherche? Étant donné la multiplication des problématiques et leur acuité, comment améliorer l'efficience des programmes de recherche?

\section{Problèmes éthiques liés à l'utilité sociale des produits agricoles ou transformés que la recherche contribue à mettre au point}

En quoi ces produits créent-ils des connaissances utiles et de la richesse sans aggraver, ou mieux en réduisant les inégalités d'accès ? Est-ce que la recherche est outillée pour répondre à des questions de cette nature?

\section{Problèmes éthiques liés à l'expertise sollicitée par les organismes de régulation}

Il y a lieu de s'interroger sur la nécessité pour la recherche agronomique française de s'impliquer dans l'élaboration, la mise au point et la validation de normes techniques ou d'outils de régulation publique, supranationale, ou privée dans le cadre des certifications. Il y a matière à identifier les domaines critiques dans lesquels elle devrait intervenir pour porter la voix des sans voix et atténuer, voire neutraliser, l'impact des groupes d'influence.

\section{Problèmes éthiques liés aux liens entre les entreprises agro-alimentaires et les équipes de recherche}

Un nombre croissant d'entreprises agroalimentaires financent des équipes de recherche et des fondations. Il y a lieu de $s^{\prime}$ interroger sur certains de ces partenariats, leurs objectifs et leur impact sur l'image de la recherche publique. Comment avoir une réflexion personnelle et collective, pour que les impératifs économiques de court terme ne se substituent pas à la légitimation éthique?

\section{Les pistes d'actions pour la recherche}

Pour tenter d'apporter une réponse aux différentes questions éthiques mentionnées, les actions mériteraient d'être orientées dans un sens qui vise à :

- Mettre en place des réflexions collectives sur les valeurs des institutions, les objectifs des travaux et leurs impacts ainsi que des formations à la complexité des systèmes alimentaires.

- Maintenir une vigilance sur la diversité des modèles alimentaires, sur les besoins des groupes les plus défavorisés et promouvoir des modèles de consommation plus soutenables.

- Mener, au sein des projets de recherche, une réflexion sur l'impact des travaux sur l'économie, les écosystèmes, les ressources, et y inscrire une réflexion sur les politiques publiques en matière alimentaire et de santé.

- Partager équitablement les connaissances scientifiques et la propriété intellectuelle dans la coopération Nord-Sud.

- Informer et alerter les décideurs publics à la fois sur les faits et tendances observés et les incertitudes.

- Participer aux débats de la société civile, à l'éducation et à l'information des consommateurs.

- Renforcer et promouvoir l'implication des acteurs des sociétés rurales et urbaines dans les différents stades de déroulement des processus de recherche relatifs à l'alimentation.

- Ouvrir un débat sur la gouvernance des systèmes alimentaires entre acteurs privés et publics.

\section{Conflits d'intérêts : aucun}

\section{RÉFÉRENCES}

CESE. Commerce éthique et dispositifs visant à apporter une garantie aux consommateurs. Avis exploratoire. REX/196. 2005.

Comité consultatif commun d'éthique pour la recherche agronomique. Avis sur "Sécurité alimentaire et modèles de consommation alimentaire ». INRA et CIRAD, mars 2010.

Chabrol D. Manger, un acte culturel. Ceras revue Projet $2008 ; 307$.

Daviron B. Hausse des prix et sécurité alimentaire mondiale. Les ruptures nécessaires. Perspective 2012, 14.

CNA. Avis sur la traçabilité des denrées alimentaires. Avis $\mathrm{n}^{\circ} 28$ adopté à l'unanimité le 28 juin 2001.

Esnouf C, Russel M, Bricas N (coord.). Pour une alimentation durable - Réflexion stratégique duALIne. Paris : Ed. Quae, 2011.

FAO. Consultation d'experts FAO sur la sécurité sanitaire des aliments: science et éthique. Rome, 3-5 septembre 2002.

$\mathrm{FAO}$. Ethique et intensification agricole durable. Collection FAO: questions d'éthique, 2004.

FAO. Sécurité alimentaire. Notes d'orientation, 2006, No 2.

FAO. L'état de l'insécurité alimentaire dans le monde. Crises économiques - répercussions et enseignements. Rome, 2009.

FAO. L'état de l'insécurité alimentaire dans le monde. Comment la volatilité des cours internationaux porte-t-elle atteinte à l'économie et à la sécurité alimentaire des pays ? Rome, 2011.

Fischler C, Masson E. Manger. Français, Européens, Américains face à l'alimentation. Paris : Ed. Odile Jacob, 2007.

Hartlieb S, Jones B. Humanising Business Through Ethical Labelling: Progress and Paradoxes in the UK. Journal of Business Ethics, 2009, 88 : 583-600.

IAASTD. Agriculture at Crossroads. Washington : Island Press, 2009a.

IAASTD. Agriculture at a Crossroads. Résumé à l'intention des décideurs, Rapport global. Washington, Island Press, 2009b.

Laisney C, Soyeux A. La consommation alimentaire à l'épreuve de la crise. Ministère de I'alimentation, de l'agriculture et des 
pêches. Analyse, Prospective et Evaluation, 2009, $10: 1-4$.

McClain-Nhlapo C. Implementing a Human Rights Approach to Food Security. IFPRI, 2004, 2020 Africa Conference Brief 13.

OMS. Obésité et surpoids. Aide-mémoire, 2006, 311.
OPECST. Rapport sur les nouveaux apports de la science et de la technologie à la qualité et à la sûreté des aliments, par M. Claude Saunier, Sénateur. Enregistré à la Présidence de l'Assemblée Nationale le 14 avril 2004.

Poulain JP. Manger aujourd'hui. Attitudes, normes et pratiques. Paris : Ed. Privat, 2002.
Sicard D. L'alibi éthique. Paris : Ed. Plon, 2006.

Sen AK. Poverty and Famines: An Essay on Entitlement and Deprivation. Oxford : Clarendon Press, 1981.

Sen AK. Repenser l'inégalité. Paris : Ed. du Seuil, 2000. 